\title{
Role of Ultrasonography as a Prognostic Tool for the Treatment of Idiopathic Clubfoot by Ponseti Technique: A Systematic Review of Literature
}

\author{
${ }^{1}$ Vishal Kumar, ${ }^{2}$ Nirmal R Gopinathan, ${ }^{3}$ Mandeep S Dhillon, ${ }^{4}$ Prasoon Kumar, ${ }^{5}$ Aman Hooda
}

\begin{abstract}
Introduction: Modern nonoperative treatment of idiopathic clubfoot consists of the Ponseti technique of manipulation and serial casting. Most patients have an excellent long-term outcomes with minimal pain or disability; however, some patients fail to respond or relapse after initial correction. The challenge is to identify such probable resistant or failed cases, and the factors that determine the same. Ultrasonography (USG) is being explored for the diagnosis and follow-up of clubfoot cases.
\end{abstract}

Research question: Does USG help in prognosticating the effectiveness of Ponseti method in clubfoot correction?

Materials and methods: Based on a PubMed search, articles related to usage of USG as a tool to prognosticate the Ponseti method were evaluated over a period of last 10 years. Five studies were identified and assessed. Based on this evaluation, parameters were extracted that could reliably indicate the prognosis.

Conclusion: Ultrasonography seems to be a cheap and effective tool to identify and calculate factors like tarsal dysplasia, talonavicular (TN) angle, talocuneiform angle (TCu), and the medial malleolus navicular distance (MMND) that could be evaluated before starting treatment and serially during its course. A low MMND and TN angle or a dysplastic talus indicates stiffness and poor prognosis. A serial increase in MMND and TN angle indicates adequate maintenance of correction. So, these parameters measured by USG could be used to prognosticate the effectiveness of the Ponseti method.

Keywords: Clubfoot, Ponseti, Prognosis, Talonavicular, Ultrasonography.

How to cite this article: Kumar V, Gopinathan NR, Dhillon MS, Kumar P, Hooda A. Role of Ultrasonography as a Prognostic Tool for the Treatment of Idiopathic Clubfoot by Ponseti Technique: A Systematic Review of Literature. J Foot Ankle Surg (Asia-Pacific) 2018;4(3):35-38.

Source of support: Nil

Conflict of interest: None

\footnotetext{
${ }^{1}$ Assistant Professor, ${ }^{2}$ Associate Professor, ${ }^{3}$ Professor and Head, ${ }^{4,5}$ Senior Resident

${ }^{1-5}$ Department of Orthopaedics, Postgraduate Institute of Medical Education and Research, Chandigarh, India

Corresponding Author: Prasoon Kumar, Senior Resident Department of Orthopaedics, Postgraduate Institute of Medical Education and Research, Chandigarh, India, Phone: +919781002142, e-mail: drprasoonksingh@gmail.com
}

\section{INTRODUCTION}

Treatment of idiopathic clubfoot consists of early initiation of manipulation and serial casting. ${ }^{1,2}$ Regular followup and bracing is advisable till 5 years of age to prevent such recurrence. ${ }^{2}$ Despite an established technique, inadequate correction and recurrence is a common problem faced by healthcare providers. The main goal of clubfoot treatment is to accomplish a normal looking, pain-free, plantigrade, and functional foot. Treatment of clubfoot remains incomplete as there is no prognostic marker available to guide treatment. Improvement in clinical (Pirani and Dimeglio scoring) scores has been used as an objective parameter to assess correction during casting and the need for tenotomy. Many patients fail to respond to Ponseti method, or undergo relapse, requiring further surgical intervention. ${ }^{3,4}$ There is $7 \%$ incidence of resistant clubfoot, and around 14\% recurrence/relapse even after initial successful correction obtained with Ponseti technique. ${ }^{5,6}$ The present major challenge is to identify these cases where conventional conservative treatment is inadequate. However, the related factors largely go unidentified and Ponseti method is applied universally in all new cases.

The use of noninvasive imaging modalities to correlate the predictive factors with the clinical response obtained to serial casting is being continuously evaluated. ${ }^{7,8}$ Ultrasonography is a common modality that has been on a surge in recent past in prenatal diagnosis and further evaluation in clubfoot., ${ }^{9,10}$

In our institute, work has been done to establish treatment protocols and assess the role of USG to evaluate parameters that could predict the outcome of this method and apply alternative treatment for such cases. ${ }^{11-13}$ Furthering the cause, the present study aims to identify such ultrasonic parameters by an extensive search of recent literature and assess the role and efficacy of USG to prognosticate the outcome of the Ponseti treatment of clubfoot.

\section{MATERIALS AND METHODS}

A thorough PubMed search was made with mixing of keywords ("Clubfoot" [Mesh] OR "CTEV" [All], "Ultrasonography" [Mesh] OR "USG" [All] and "treatment

(This article was given best paper award at 2016 conference) 
prognosis" [All]) on 14/10/2017. Literature search was limited to last 10 years, between 2007 and 2017. A total of 184 hits were obtained. All abstracts of the articles were read by three of the authors, leading to exclusion of 179 papers. No level 1 evidence articles on the subject were found. Papers unrelated to conservative treatment, related to prenatal diagnosis and surgical treatments, review articles, and animal studies were excluded. Neurological clubfeet was also excluded. A total of five studies were included. Two of the studies are from our institute.

\section{RESULTS}

Several studies have described the use of sonography in clubfoot. Tolat et $\mathrm{al}^{10}$ discussed the abnormal positions of the talus and navicular in pathology of clubfoot but they made no attempt to correlate their findings with radiographs or clinical classification of severity.

In our literature search, we identified five relevant studies, all of which assessed the ultrasonic parameters in clubfoot (Table 1). Chandrakanth et $\mathrm{al}^{11}$ highlighted the role of tarsal dysplasia in predicting the response to casting, with increasing severity of tarsal dysplasia requiring prolonged casting. They included 25 infants with unilateral idiopathic clubfoot without any history of treatment. An initial USG was done before commencing the serial casting. Three standard planes were used to measure maximum length of talus, calcaneus, and navicular bones. Ratios of their maximum lengths to bones in normal feet were calculated and taken as the amount of dysplasia. The number of casts required and the need for surgical correction were used as parameters to assess outcomes. They found a significant correlation between number of casts required and dysplasia of talus. The authors also determined the dysplasia of each tarsal bone in comparison to each other in both feet of every patient. This was done to evaluate whether or not all the tarsal bones were proportionately dysplastic. Ratio of the talus length to the calcaneum and navicular was used to evaluate this relative dysplasia. The results showed that relative dysplasia of talus in comparison to calcaneum is correlated to the number of casts required. Therefore, in the affected feet if talus is more dysplastic than calcaneum, it signifies inferior outcomes. They concluded that tarsal bone dysplasia quantified by USG can be a promising tool used for prognostic indicator in idiopathic clubfoot.

Another study by Gupta et $\mathrm{al}^{12}$ evaluated the role of $\mathrm{TN}$ angle in clubfoot, documented during the course of correction using the Ponseti method, and noted it to be a reliable prognostic indicator. They included 26 infants with unilateral clubfoot and treated them by this method. They prospectively evaluated the outcomes by clinical Dimeglio and Pirani scores. Ultrasonography was done to measure the TN angle at the beginning of the treatment, after mid correction, and at the end of complete correction; they also noted total number of casts required.

They established a negative correlation between change in TN angle during course of treatment and the clinical scores and number of casts required for deformity correction. It was concluded that change in TN angle was the best predictor of the outcome and it could easily be evaluated using the ultrasound; this is thus an inexpensive, easily reproducible, and objective method to prognosticate outcomes of clubfeet correction using the Ponseti method. ${ }^{12}$

Desai et $\mathrm{al}^{14}$ conducted a prospective study on 32 clubfeet in 26 patients and used USG with the coronal medial projection at start and end of Ponseti treatment. They used ultrasonographic method to measure MMND and $\mathrm{TCu}$ and correlated it with the prognosis in clubfoot. The normal feet in unilateral cases were taken as controls. They found a significant rise in the MMND

Table 1: Review of literature

\begin{tabular}{|c|c|c|c|c|c|c|}
\hline Authors & Year & $\begin{array}{l}\text { Level of } \\
\text { study }\end{array}$ & $\begin{array}{l}\text { No. of } \\
\text { feet }\end{array}$ & $\begin{array}{l}\text { USG } \\
\text { parameters }\end{array}$ & Study conclusion & Our inference \\
\hline 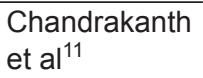 & 2015 & 2 & 25 & TL, CL, NL & $\begin{array}{l}\text { Talus dysplasia could cause prolonged } \\
\text { casting and worse outcome }\end{array}$ & $\begin{array}{l}\text { Shortening of tarsal bones like talus } \\
\text { indicates stiffer feet and poor outcome }\end{array}$ \\
\hline Gupta et al ${ }^{12}$ & 2012 & 2 & 26 & TN angle & $\begin{array}{l}\text { Negative correlation b/w change in } \\
\text { TN angle and clinical scores and no. } \\
\text { of casts }\end{array}$ & $\begin{array}{l}\text { Low TN angle and less change over } \\
\text { the course predicts worse deformity, } \\
\text { prolonged treatment, and poorer } \\
\text { outcome }\end{array}$ \\
\hline Desai et al ${ }^{14}$ & 2008 & 4 & 32 & $\begin{array}{l}\text { MMN, TCu } \\
\text { angle }\end{array}$ & $\begin{array}{l}\text { Low MMND and high TCu angle } \\
\text { make the feet stiffer and outcome } \\
\text { poorer }\end{array}$ & $\begin{array}{l}\text { Small MMN distance and increased } \\
\text { TCu angle can predict unfavorable } \\
\text { outcome }\end{array}$ \\
\hline $\begin{array}{l}\text { El-Adwar and } \\
\text { Taha Kotb }^{15}\end{array}$ & 2010 & 2 & 25 & $\begin{array}{l}\text { MMN, NAL, } \\
\text { TL, CC } \\
\text { distance }\end{array}$ & $\begin{array}{l}\text { Increased CC distance with } \\
\text { malalignment causes poor prognosis. } \\
\text { MMND and talus length are useful }\end{array}$ & $\begin{array}{l}\text { Small MMND and increased CC } \\
\text { distance are unfavorable for good } \\
\text { outcome. Talus shortening indicates } \\
\text { worse results }\end{array}$ \\
\hline $\begin{array}{l}\text { Miron and } \\
\text { Grimard }^{16}\end{array}$ & 2015 & 3 & $\mathrm{~N} / \mathrm{A}$ & $\begin{array}{l}\text { TC angle, } \\
\text { MMN }\end{array}$ & $\begin{array}{l}\text { Low } \mathrm{TC} \text { and } \mathrm{MMN} \text { denote severity of } \\
\text { varus }\end{array}$ & $\begin{array}{l}\text { Small MMN and TC angles are } \\
\text { unfavorable for Ponseti method }\end{array}$ \\
\hline
\end{tabular}

TiC: Tibiocalcaneal; TL: Talar length; CL: Calcaneal length; NL: Navicular length; NAL: Navicular alignment; MMND: Medial

malleolus navicular distance; TC: Talocalcaneal angle; CC: Calcaneocuboid; TCu: Talocuneiform; N/A: Not available 
with improvement of $\mathrm{TCu}$ at the end of treatment as documented by USG. All feet were clinically corrected, though five of them showed a spurious correction; they were considered cosmetically and functionally good, but showed a midfoot break on USG. This spurious correction was due to the navicular not reducing completely over the talar head. The increase in MMND in these five cases was less than in the anatomically corrected feet and was statistically significant. Additionally, the pretreatment values of the MMND were less, signifying that these feet were stiffer and more severely deformed, and hence had the potential for an inferior outcome. The TCu angle in these five feet also showed more increase, indicating the incongruence of the naviculo-cuneiform joint, leaving the navicular unreduced. The authors concluded that MMND could be a useful parameter to recognize the pretreatment stiffness of a clubfoot. Shorter MMND as measured by USG could be a guide to look for alternative treatment methods.

El-Adwar and Taha Kotb ${ }^{15}$ attempted to establish a correlation between various USG parameters like MMND, talar length, calcaneocuboid (CC) joint congruency, and TN joint reduction with the Pirani score. They followed 17 infants with 25 clubfeet treated with the Ponseti method, assessing the above-mentioned variables. They observed a pretreatment negative correlation between the clinical midfoot score and the MMND. They also observed negative correlation, posttreatment, between the talar length and the midfoot Pirani score, and the MMND with the hindfoot score. The feet showing recurrence had increased CC distance. They concluded that USG can alert a surgeon to the feet that may be prone to recurrence or relapse, based on these correlations. Small MMND and small length of the talus could indicate rigid feet and poor prognosis.

Miron and Grimard ${ }^{16}$ evaluated various foot deformities in infants with USG and suggested their usage as an additional tool to clinical examination for assessing the severity of the deformity and treatment efficacy of the Ponseti method for clubfeet. They used five scanning planes-medial coronal, medial coronal dual, dorsal sagittal, lateral coronal, and posterior sagittal (in neutral and dorsiflexion). The primary abnormality in clubfoot is the TN malalignment with medial subluxation of navicular over talar head, along with decreased MMND. The authors measured the TC angles with USG, which correlated with the angles measured by the anteroposterior X-rays. They concluded that lower TC angles depict severity of the hindfoot varus. The authors recommend usage of USG at the beginning and in the course of the serial casting. They state that USG can help even in decision regarding need of Achilles tenotomy. Small MMN and low TC angles were the parameters identified to be useful for prognostic role.

\section{DISCUSSION}

The five studies evaluated in this review identified several angles and parameters that could be used in prognosticating Ponseti method. There were no level 1 studies available on this topic. Three of the studies were level $2,^{11,12,15}$ while 1 each were of level 3 and $4 .^{14,16}$ Four studies state the number of feet included while one does not. ${ }^{16}$ The relative number of feet though is not large in any study ranges from 25 to 32 feet.

Ultrasonography is an objective, cheap, and inexpensive modality to evaluate response to casting. The small bones of a neonate are cartilaginous and can be well visualized on USG, making it superior to conventional radiographs. Utilization of adequate planes to do the USG is important with the coronal medial plane being most important, as it measures the navicular alignment with the talus.

Talonavicular malalignment is the most serious component of the deformity, requiring correction, with other major abnormalities including medial angulation of talar head and neck and the CC subluxation.

Importance of TN angle was assessed by Gupta et $\mathrm{al}^{12}$ who found a negative correlation between the change in this angle measured serially during the course of treatment with the clinical scores and number of casts required. This shows that if during the treatment there is minimal change or increase in this angle, the treatment gets prolonged and the deformity worsens, increasing the clinical Dimeglio and Pirani scores.

Medial malleolus navicular distance is another parameter measured by USG that is identified to be very useful in treatment of clubfeet. ${ }^{14-16}$ It is the distance between the tip of medial malleolus and the medial end of the navicular in millimeters. Desai et $\mathrm{al}^{14}$ found that lesser pretreatment distance makes the feet stiffer. There is an increase in MMND during the serial manipulation and it becomes significantly higher with deformity correction. Inadequate increase can cause spurious correction. El-Adwar and Taha Kotb ${ }^{15}$ and Miron and Grimard ${ }^{16}$ also found low MMND to be a very useful predictor of poor outcome with higher clinical scores.

Tarsal dysplasia is a coexisting finding in clubfeet. The severity of this can be identified by USG and can be used to prognosticate the outcome. Chandrakanth et $\mathrm{al}^{11}$ found that talus shortening or dysplasia is related to stiffer feet and requires more number of casts for deformity correction (Table 1). Similar correlation was reported by ElAdwar and Taha Kotb. ${ }^{15}$ Even if the talus is relatively shorter than the calcaneum or navicular, as compared with the normal side, the chances of inferior outcomes are high. ${ }^{11,15}$ 
Other angles like TCu or CC distance can also be measured by USG and their increased value could indicate unsuitable outcomes. ${ }^{14,15}$

\section{CONCLUSION}

The application of USG in the evaluation of clubfoot to determine prognosis and treatment protocol is evolving into a cost-effective and efficient method to prognosticate the outcome of clubfoot treatment by Ponseti method.

However, none of the studies have evaluated it on a large scale. The use of USG and the timing of its application need to be standardized.

Further large-scale multicentric studies are warranted. This could help in individualizing the treatment protocol as per the requirements of each patient, taking their anatomical factors into consideration and streamlining the treatment, thus saving time and money, as well as minimizing the mental and physical agony which families undergo when the problem relapses.

\section{REFERENCES}

1. Wynne-Davies R. Genetic and environmental factors in the etiology of talipes equinovarus. Clin Orthop Relat Res 1972 May;84:9-13.

2. Bridgens J, Kiely N. Current management of clubfoot (congenital talipes equinovarus). BMJ 2010 Feb;340:c355.

3. Cooper DM, Dietz FR. Treatment of idiopathic clubfoot. A thirty-year follow-up note. J Bone Joint Surg Am 1995 Oct;77(10):1477-1489.

4. Dobbs MB, Rudzki JR, Purcell DB, Walton T, Porter KR, Gurnett CA. Factors predictive of outcome after use of the Ponseti method for the treatment of idiopathic clubfeet. J Bone Joint Surg Am 2004 Jan;86-A(1):22-27.

5. Carroll NC, McMurtry R, Leete SF. The pathoanatomy of congenital clubfoot. Orthop Clin North Am 1978 Jan;9(1): 225-232.
6. Ippolito E. Update on pathologic anatomy of clubfoot. J Pediatr Orthop B 1995;4(1):17-24.

7. Byron-Scott R, Sharpe P, Hasler C, Cundy P, Hirte C, Chan A, Scott H, Baghurst P, Haan E. A South Australian populationbased study of congenital talipes equinovarus. Paediatr Perinatal Epidemiol 2005 May;19(3):227-237.

8. Abdelgawad AA, Lehman WB, van Bosse HJ, Scher DM, Sala DA. Treatment of idiopathic clubfoot using the Ponseti method: minimum 2-year follow-up. J Pediatr Orthop B 2007 Mar;16(2):98-105.

9. Lin J, Fessell DP, Jacobson JA, Weadock WJ, Hayes CW. An illustrated tutorial of musculoskeletal sonography: part I, introduction and general principles. AJR Am J Roentgenol 2000 Sep;175(3):637-645.

10. Tolat V, Boothroyd A, Carty H, Klenerman L. Ultrasound: a helpful guide in the treatment of congenital talipes equinovarus. J Pediatr Orthop B 1995 Feb;4(1):65-70.

11. Chandrakanth U, Sudesh P, Gopinathan N, Prakash M, Goni VG. Tarsal bone dysplasia in clubfoot as measured by ultrasonography: can it be used as a prognostic indicator in congenital idiopathic clubfoot? A prospective observational study. J Pediatr Orthop 2016 Oct-Nov;36(7):725-729.

12. Gupta N, Sudesh P, Prakash M, Tripathy SK, Dhillon MS. Is sonographic calculation of Talonavicular angle during Ponseti correction of clubfoot a reliable prognostic indicator? J Postgrad Med Edu Res 2012 Apr;46(4):190-195.

13. Mootha AK, Saini R, Krishnan V, Bali K, Kumar V, Dhillon MS. Management of idiopathic clubfoot by the Ponseti technique: our experience at a tertiary referral centre. J Orthop Sci 2011 Mar;16(2):184-189.

14. Desai S, Aroojis A, Mehta R. Ultrasound evaluation of clubfoot correction during Ponseti treatment: a preliminary report. J Pediatr Orthop 2008 Jan-Feb;28(1):53-59.

15. El-Adwar KL, Taha Kotb H. The role of ultrasound in clubfoot treatment: correlation with the Pirani score and assessment of the Ponseti method. Clin Orthop Relat Res 2010 Sep;468(9):2495-2506.

16. Miron MC, Grimard G. Ultrasound evaluation of foot deformities in infants. Pediatr Radiol 2016 Feb;46(2):193-209; quiz 190-192. 